\title{
Contaminación del aire en la Ciudad Autónoma de Buenos Aires: El riesgo de hoy o el cambio climático futuro, una falsa opción
}

\author{
Air contamination in the Autonomous City of Buenos Aires: \\ the current risk or future climate change, a false option
}

Rosana Abrutzky ${ }^{1}$

Laura Dawidowski ${ }^{2}$

Ana Murgida ${ }^{3}$

Claudia Eleonor Natenzon ${ }^{3}$
${ }^{1}$ Universidad Nacional de General San Martín. Av. 25 de Mayo y Francia, General San Martín. B1650HMP Buenos Aires Bs. As. Argentina. rabrutzky@gmail.com ${ }^{2}$ Comisión Nacional de Energía Atómica.

${ }^{3}$ Programa de Investigaciones en Recursos Naturales y Ambiente, Facultad de Filosofía y Letras, Universidad de Buenos Aires.

\begin{abstract}
Based on the theoretical framework of environmental risk, this article discusses the management of air quality in the Autonomous City of Buenos Aires in relation to current and potential impacts of toxic gases and global climate change on the health of the population. Information on historical and current management of the air was linked to the results of the South American Emissions, Megacities and Climate research project to assess danger, exposure, vulnerability and uncertainty as the dimensions of risk. By contextualizing public policies developed in recent decades on this subject, it was possible to identify emerging configurations of risk and uncertainties as accelerators of social vulnerability. On the one hand, the fact that there is a positive correlation between mortality, changes in temperature and air pollution was confirmed. On the other hand, it became clear that there is a disconnect between air quality management and health care management, while limitations were found in the proposed mitigation measures relating to emissions of greenhouse gases produced by fuel, revealing uncertainties regarding their efficacy.
\end{abstract}

Key words Pollution, Risk, Climate change, Public health, Autonomous City of Buenos Aires
Resumen Desde el marco teórico del riesgo ambiental, el presente artículo aborda la gestión de la calidad del aire en la Ciudad Autónoma de Buenos Aires en relación con los impactos actuales y potenciales de los gases tóxicos y del cambio climático global sobre la salud de la población. Se vinculó información sobre gestión histórica y actual del aire con los resultados del proyecto de investigación South American Emissions, Megacities and Climate para evaluar peligrosidad, exposición, vulnerabilidad e incertidumbre como dimensiones del riesgo. Al contextualizar las politicas públicas desarrolladas en las últimas décadas sobre este tema, fue posible identificar configuraciones de riesgo y de incertidumbres emergentes, amplificadoras de la vulnerabilidad social. Por una parte se confirmó que existe una correlación positiva entre la mortalidad, los cambios en la temperatura y en la contaminación del aire. Por otra se constató la desvinculación entre la gestión de la calidad del aire y la gestión de salud, a la vez que se encontraron limitaciones en las medidas de mitigación propuestas en relación a las emisiones de gases efecto invernadero producidas por los combustibles, señalando las incertidumbres respecto a su eficacia.

Palabras clave Contaminación atmosférica, Riesgo, Cambio climático, Salud pública, Ciudad Autónoma de Buenos Aires 


\section{Introducción}

La preocupación por la composición atmosférica y su relación con la salud, generalizada a partir de los grandes episodios de contaminación urbana de mediados del siglo pasado, ha cobrado un impulso importante y se ha complejizado en las últimas décadas a raíz de la constatación del cambio climático global. Un importante volumen de bibliografía sobre el tema se refiere a estudios realizados en los países centrales; mientras que los países en desarrollo, y América Latina en particular, poseen un creciente cuerpo de datos, análisis y equipos de investigación que posibilitan realizar análisis propios acerca de los fenómenos vinculados a la calidad del aire, sus causas y consecuencias $^{1-5}$. Sin embargo, raramente los resultados de estos estudios son incorporados por la gestión pública ambiental, y menos aún por aquella vinculada con la salud.

El trabajo que aquí se presenta muestra los resultados del Proyecto SAEMC-South American Emissions, Megacities and Climate, financiado por el IAI-Instituto Inter Americano para el Estudio del Cambio Global entre 2006 y 2011, en el cual colaboraron diversas instituciones de investigación científico-técnicas de América Latina y donde se combinaron saberes provenientes de diversas ciencias naturales y sociales. Los resultados de este trabajo interdisciplinario arrojaron luz sobre diversos aspectos tanto de la calidad del aire como de su gestión las ciudades de Buenos Aires, Bogotá, Santiago de Chile, Lima, México y San Pablo ${ }^{6-9}$. En cada una de ellas se buscó conocer las características de la composición del aire, su relación con los problemas de salud y su impacto en el fenómeno del cambio global. En el presente artículo nos interesa retomar los resultados obtenidos para la CABA - Ciudad Autónoma de Buenos Aires, analizándolos desde la teoría del riesgo para alumbrar las formas en que socialmente se construye la calidad del aire como problema, y las decisiones que se implementan en consecuencia.

Para gestionar el riesgo es necesario conocer sus dimensiones constitutivas ${ }^{10}$ que incluyen la peligrosidad inherente a los fenómenos (espontáneos o manipulados técnicamente), la vulnerabilidad de los grupos sociales involucrados, la exposición correspondiente a cada configuración territorial y la incertidumbre emergente de cada una de las anteriores. En particular, el análisis de la vulnerabilidad social permite comprender el nivel de dificultad o las capacidades que tendrá cada grupo social para hacer frente y recuperarse autónomamente luego de cualquier afectación. Según Carlos Filgueira ${ }^{11}$, ella se refiere tanto a los atributos individuales o domésticos como a la estructura de oportunidades que el contexto socioeconómico brinda a individuos o familias. Precisamente sobre este último punto es que se pone aquí la atención, considerando que el accionar de las instituciones juega un rol central en la estructura de oportunidades, con influencia directa tanto en la disminución o la amplificación de las condiciones vulnerables como la generación de crecientes incertidumbres.

La consideración de este caso de contaminación del aire urbano es un aporte a los estudios de ciudades en países de media o baja renta, justamente los que menos se han realizado ${ }^{12}$. Se busca poner en evidencia cómo se construye socialmente el riesgo, vinculando los avances del saber experto con las reales condiciones de la gestión, analizando cómo una administración municipal aborda la cuestión de la contaminación del aire y lo articula o no, con los objetivos y los compromisos relativos al cambio climático. El caso permite poner de manifiesto la relevancia para la salud tanto de los eventos agudos producidos por fenómenos extremos (por ejemplo, inundaciones, deslizamientos o huracanes) como de los eventos crónicos (por ejemplo, contaminación atmosférica, hídrica o edáfica); y que en muchas oportunidades ambos se superponen, aumentando el riesgo y los efectos negativos sobre la salud por combinación de fuentes peligrosas con personas vulnerables, expuestas a esos peligros. Sin embargo, al analizar las políticas y la gestión pública puede verse que los riesgos son tratados de manera fragmentada, aisladamente, sin reconocer su intrínseca complejidad.

\section{Marco teórico-metodológico}

La sociedad moderna es una "sociedad del riesgo" 13,14 , donde la ocurrencia de situaciones accidentales o imprevistas son resultado de nuestras propias actividades o decisiones, y no obra divina, de la fortuna o la fatalidad.

El riesgo es una potencialidad, algo que puede llegar a ocurrir. Si este potencial se concreta, el resultado es desastroso. Por eso algunos autores señalan que la catástrofe es la actualización del riesgo ${ }^{15}$. Cuando hablamos de riesgo inequívocamente estamos aludiendo a un proceso social de múltiples determinaciones y alto grado de complejidad ${ }^{16}$. Se habla de riesgo cuando es posible cuantificar, establecer una probabilidad. Cuando no se puede establecer una probabilidad respec- 
to a qué va a ocurrir ya no se tiene riesgo sino incertidumbre y el futuro es incierto. Así, una catástrofe (como algo "extraordinario") concreta el potencial de determinado riesgo, poniendo en tensión y desenmascarando las características existentes ("normales") del proceso de desarrollo en una dada sociedad.

Caracterizar el riesgo permite tomar medidas para evitar potenciales catástrofes, para lo cual es necesario estudiar sus cuatro dimensiones constitutivas: peligrosidad, vulnerabilidad, exposición e incertidumbre ${ }^{10,17}$.

La peligrosidad se refiere al potencial peligroso inherente a los fenómenos espontáneos o manipulados, cualquiera sea su grado de artificialidad (en el sentido de "artefacto", hecho con arte, hecho por el hombre, incluyendo en esto a lo "tecnológico"). Un fenómeno sólo adquiere la condición de peligroso cuando su ocurrencia se da o se prevé en un espacio ocupado por una determinada sociedad. La peligrosidad puede ser tomada de distintas maneras: la peligrosidad científico-técnica en base a lo que dicen los expertos en el marco de la ciencia normal; o la peligrosidad percibida, basada en las representaciones sociales, es decir, según es identificada por distintos sectores de la población.

La vulnerabilidad es el conjunto de características de una persona o un grupo que determina su capacidad para anticiparse, sobrevivir, resistir y recuperarse del impacto de un peligro", posibilidad que tienen de enfrentarlo y recuperarse ${ }^{18,19}$. La vulnerabilidad social se define por las condiciones de una sociedad previas a la ocurrencia de eventos catastróficos, que la predisponen para sufrir o evitar daños de todo tipo; considera la capacidad de adaptación y de reacción. Ella es diferencial para cada grupo, y determina el nivel de dificultad o las capacidades que tendrá cada uno de ellos para recuperarse autónomamente luego de la afectación.

La exposición refiere a la distribución geográfica de lo que podría ser materialmente afectado, población y bienes. Es una consecuencia de la interrelación entre peligrosidad y vulnerabilidad, y -a la vez- incide sobre ambas. Esta componente se expresa territorialmente como construcción histórica que entrelaza los procesos físico-naturales con las relaciones socioeconómicas, configurando determinados usos de suelo y distribución de infraestructura, asentamientos humanos, servicios públicos, etc.

Peligrosidad, vulnerabilidad y exposición constituyen el riesgo. El disparador (la peligrosidad), plantea desafíos variados según sea el tipo de proceso involucrado, lo que influye en una exposición también diferencial. La vulnerabilidad también es heterogénea y se presenta como un común denominador.

Cada dimensión trae aparejados su propia incertidumbre a la vez que presenta aspectos que aún no han sido caracterizados o, incluso, identificados. Sin embargo, son cuestiones que requieren una respuesta urgente por los valores que están en juego: bienes, vidas, sociedades, cultu$\operatorname{ras}^{20}$. El abordaje de la incertidumbre que crece a expensas del desconocimiento en cada una de las dimensiones, debe realizarse a partir de la trama de las relaciones sociales, de la toma de decisiones y de la subjetividad. La falta de evaluaciones expertas y la ausencia de respuestas y soluciones políticas democráticas aumentan la brecha entre las certezas y los desconocimientos acerca de la magnitud de los riesgos existentes, y suele provocar diversas reacciones tales como miedo, negligencia, confusión o descreimiento.

El sistema científico-técnico (el "saber experto") ha realizado propuestas para la gestión de catástrofes. Sin embargo, a menudo este saber resulta ajeno a los contextos -en este caso, el urbano-, configurando un planteo teórico-ideal que no toma en cuenta las reales circunstancias en las que pueden evitarse daños. De esta manera, las instituciones no realizan una gestión del riesgo preventiva sino acciones coyunturales en la emergencia.

En función de este marco conceptual se analizará aquí la situación de la Ciudad de Buenos Aires relevando la gestión de la calidad del aire histórica y actual en base a bibliografía y documentación oficial, y se incorporarán los resultados producidos por el proyecto SAEMC para ilustrar la relación entre la existencia de datos, las incertidumbres y la toma de decisiones en lo referido a los riesgos ambientales vinculados con los procesos atmosféricos. La consideración de este caso urbano permitirá conocer la forma en que se construye socialmente el riesgo, vinculando lo propuesto desde el saber experto con las condiciones de gestión.

\section{Caracterización de la CABA}

La Ciudad Autónoma de Buenos Aires es la capital de la República Argentina. Ubicada en el sur de Sudamérica, con una superficie de $203 \mathrm{~km}^{2}$ totalmente urbanizados, tiene una población de poco menos de 3 millones de habitantes ${ }^{21}$.

$\mathrm{Al}$ este y noreste limita con el estuario del río de la Plata, mientras que por el norte, oeste y sur 
está rodeada por 32 municipios de la provincia de Buenos Aires, completa o parcialmente urbanizados, con los cuales comparten la misma cuenca atmosférica. La ciudad se asienta sobre un área plana, conformada por dos unidades geomorfológicas: la llanura pampeana y la planicie estuári$\mathrm{ca}$, con alturas que van de los 0 a los $27 \mathrm{msnm}^{22}$.

A pesar de los altos niveles de emisión de contaminantes procedentes de fuentes tanto fijas como móviles, las condiciones atmosféricas de la ciudad y su topografía evitan su acumulación y favorece su dispersión sobre una gran área que incluye el Océano Atlántico. Vientos de diferentes direcciones soplan todo el año, los más frecuentes provienen del noreste, en invierno pueden irrumpir sistemas frontales desde el sur, mientras que entre otoño y primavera se pueden producir vientos intensos del sector S-SE (denominados "sudestada") que ocasionan crecidas e inundaciones en la zona ribereña ${ }^{23}$.

En la región central de Argentina donde se ubica la CABA se ha observado un aumento en las temperaturas mínimas, cambios en la duración de las estaciones, un aumento en los promedios de precipitaciones - especialmente evidente a partir de las décadas del '60 y el '70 - y una tendencia al aumento de eventos de precipitaciones extremas ${ }^{24}$. Durante el siglo XX, el nivel medio del río de la Plata en la ciudad de Buenos Aires aumentó unos $17 \mathrm{~cm}$.; el incremento más significativo fue durante la década de 1970. Este cambio estaría asociado al incremento del nivel medio del $\operatorname{mar}^{25}$. Estas tendencias de la dinámica climática han generado consecuencias visibles (inundaciones, cambios en las alturas consideradas seguras para la construcción, erosión costera, incendios forestales y de pastizales, olas de calor) lo que permiten decir que la dinámica del clima se ha vuelto un forzante más en la producción de escenarios de riesgo.

\section{Las emisiones urbanas: caracterización de su peligrosidad}

Cualquier gestión urbana de la calidad del aire puede ser abordada desde dos enfoques. Por un lado está el que considera a los compuestos modificadores del clima de la Tierra, como el dióxido de carbono $\left(\mathrm{CO}_{2}\right)$, metano $\left(\mathrm{CH}_{4}\right)$ y óxido nitroso $\left(\mathrm{N}_{2} \mathrm{O}\right)$, entre otros, llamados en su conjunto GEI-gases de efecto invernadero. Por otro, el enfoque que considera aquellos compuestos que afectan a la salud y a la calidad de vida de la población local, como monóxido de carbono (CO), óxidos de nitrógeno (NOx), dióxido de azufre $\left(\mathrm{SO}_{2}\right)$, material particulado en suspensión (MP), compuestos orgánicos volátiles no metánicos (NMVOCs), ozono $\left(\mathrm{O}_{3}\right)$ y plomo $(\mathrm{Pb})$, entre otros. El proceso atmosférico en cuestión es complejo porque los compuestos tóxicos que afectan a la salud son a la vez precursores de gases de efecto invernadero.

En algunos casos la reducción de ambos tipos de compuestos se puede encarar de manera simultánea. Un ejemplo de esto puede ser un plan de ordenamiento vehicular que reduzca las emisiones, abarcando al mismo tiempo la reducción de GEI y de gases tóxicos. En otros casos esas medidas pueden estar contrapuestas, en el sentido de producir reducción de un tipo de compuesto y aumento de las emisiones del otro, como pasa con la sustitución de combustibles tradicionales (gasolina y diesel) por biocombustibles, cuya adopción se traduce en una reducción de gases de efecto invernadero pero que, sin las tecnologías adecuadas, incrementará la emisión de gases tóxicos. Si bien hay innumerable bibliografía respecto de las emisiones vinculadas a su uso, en general esa información es válida para tecnologías europeas o de países con hábitos de mantenimiento vehicular muy diferentes a los que se aplican en Argentina. Por esa razón, en el marco del proyecto SAEMC se buscó analizar las posibilidades de reducción con una mirada conjunta que abarque lo ambiental y lo tecnológico desde una perspectiva amplia (y no sólo aspectos económicos) incluyendo gases de efecto invernadero pero también compuestos tóxicos.

En la CABA, la calidad del aire no ha sido hasta el presente un problema de agenda pública. A pesar del alto nivel de emisiones de la ciudad, sus condiciones topográficas y atmosféricas favorables hacen que presente niveles de concentración de compuestos tóxicos menores que otros centros urbanos de características similares, y por lo general con niveles inferiores a los valores máximos permitidos en la legislación local. Sin embargo, si se consideran las normas de calidad del aire indicadas por la Organización Mundial de la Salud, en algunos casos los niveles de concentración de material particulado en suspensión y óxidos de nitrógeno registrados resultan elevados.

Del mismo modo, no existió una iniciativa para evaluar la carga de compuestos emitidos por las distintas fuentes presentes en la ciudad. Esa carencia motivó que el equipo de investigación de SAEMC desarrollara el inventario de emisiones de fuentes fijas y móviles, necesario no sólo para evaluar el rol de los distintos sectores en las emisiones, sino también como pun- 
to inicial para la modelización y pronóstico del tiempo químico en la atmósfera. Como resultado de este trabajo, se identificó que las emisiones de GEI son producidas predominantemente por las fuentes fijas, en particular por las dos centrales térmicas instaladas en la costa del Río de la Plata y por el consumo domiciliario, mientras que el transporte carretero es el principal responsable de las emisiones de los compuestos tóxicos. A su vez, dentro del sector transporte la tecnología del vehículo y el tipo de combustible determina las fuentes principales de emisión: mientras los vehículos antiguos a gasolina son los principales emisores de CO y de NMVOCs, los camiones que circulan por la ciudad son los responsables de las emisiones de material particulado, seguidos por los autobuses. Las emisiones de SO2 están vinculadas fundamentalmente al uso de combustibles líquidos en las centrales térmicas, pero también al tránsito pesado debido a los altos niveles de azufre del diesel en la Argentina ${ }^{6}$.

\section{Vulnerabilidad institucional e incertidumbre}

Desde mediados del siglo pasado, las políticas del aire de la CABA estuvieron caracterizadas por cambios de objetivos y métodos a través de las distintas gestiones. Cada cambio en el objetivo perseguido y su expresión en modificaciones de la agenda vino acompañado por cambios en el tipo de compuestos medidos, en la metodología y en los instrumentos de medición, lo que no ha contribuido a generar condiciones más salubres para la población expuesta a aire contaminado.

Si bien la CABA fue pionera en la vigilancia atmosférica, comenzando con mediciones a inicios de la década del 70, esta iniciativa disminuyó entre 1975 y 2008, período en el cual las actividades de diagnóstico de la calidad del aire fueron escasas, manteniendo sólo una estación que fue desmantelada en 2007 y agregando otra en 2005 que sigue funcionando hasta el presente. A partir del año 2009 se pudo concretar la instalación (largamente planeada) de dos nuevas estaciones, que junto con la anterior, constituyen la red de monitoreo de la CABA, donde se miden en forma automática $\mathrm{CO}$, NOx y $\mathrm{PM}_{10}$ con métodos estandarizados.

Inicialmente, en los años 70, se desarrolló un período de diálogo y acuerdos entre las agencias gubernamentales y la comunidad científica para medir gases peligrosos para la salud de la población siguiendo lineamientos propuestos por agencias internacionales. Se identificaron, así, zonas con mayor deterioro de la calidad del aire en la ciudad y se generaron medidas regulatorias para controlar la concentración de contaminantes ${ }^{26-28}$. Esta orientación política local de la gestión del aire fue perdiendo presencia en la agenda a partir de la dictadura militar y sólo se retomó parcialmente a partir de 1987, con el primer gobierno electo tras el retorno al sistema democrático.

Hacia la década de los noventa, el problema del aire se resituó en la agenda local, pero esta vez vinculado con la problemática del cambio climático global, contando con recomendaciones internacionales y con financiamiento externo para efectuar mediciones e inventariar los GEI. Las acciones locales desarrolladas en la gestión del aire de la CABA tendieron entonces, a la reducción de emisiones GEI.

Medidas nacionales vinculadas al transporte, tomadas en base a consideraciones económicas, convergieron con esta preocupación local:

a) La incorporación del GNC-gas natural comprimido como combustible de los automóviles y su amplia difusión. Esta medida fue tomada en base a aspectos económicos, por su costo menor que las gasolinas, y valorada ambientalmente por su contribución a la reducción de GEI. No obstante no se tuvo en cuenta las emisiones de compuestos tóxicos provenientes de la quema de este combustible (CH4 y N2O), ni ellas fueron evaluadas en el contexto de la calidad del aire y el posible impacto sobre la salud de quienes habitan y circulan por la ciudad.

b) El proceso de reconversión del parque automotor con estímulos crediticios. Esta medida alentó el crecimiento del parque automotor y fue sinérgica con los resultados de la política inmobiliaria, que promovía la expansión de las áreas residenciales hacia las periferias del Área Metropolitana de Buenos Aires. En consecuencia aumentaron los desplazamientos y las emisiones ${ }^{6,28,29}$.

La movilización social emergente en la crisis argentina del 2001, la necesaria apertura a procesos participativos y las presentaciones judiciales realizadas por vecinos y Organizaciones No Gubernamentales (ONG), referidas a la contaminación del aire por fuentes fijas y móviles, a la producción de ruidos y a la contaminación de los cursos de agua del área metropolitana, tuvieron como consecuencia que entre 2001 y 2004 esta mirada centrada en las emisiones GEI fue necesariamente complementada por diálogos intersectoriales para articular los problemas de salud y calidad del aire ${ }^{28}$. En estos diálogos participaron el gobierno de la ciudad y ONG locales e inter- 
nacionales. Como resultado de esta interacción, se establecieron programas de vigilancia epidemiológica. Esto quedó plasmado en leyes de la CABA: la de Calidad Atmosférica N 1357/2004; la de Regulación, Control y Gestión de Aceites Vegetales Usados $N^{\circ} 1884 / 2006$; y la de Información Ambiental No 303/2006; y en la creación del Programa de Prevención de la Contaminación y Gestión Ambiental de Residuos Urbanos y Control de la Contaminación Hídrica.

Sin embargo, este proceso no redundó en el ajuste de los mecanismos para tomar datos de salud relacionados con la contaminación del aire. Un ejemplo de ello es la falta de registro y sistematización de causas frecuentes de las consultas urgentes y los egresos hospitalarios, causas que podrían vincularse con la calidad del aire y con los sitios de exposición. En este contexto, el Gobierno de la Ciudad acordó con el organismo internacional ICLEI-Iniciativas de Gobiernos Locales para la Sustentabilidad en elaborar un inventario de emisiones GEI. Éste, que estuvo disponible en el año 2003, no hace ninguna referencia a contaminantes tóxicos de importancia local.

El enfoque en las emisiones GEI continuó prevaleciendo en las políticas ambientales del Gobierno de la Ciudad, que en 2008 ratificó la colaboración con ICLEI incorporándose la Fundación Clinton, promotora de la iniciativa 40 Ciudades para la Protección del Clima - C40 ${ }^{30}$. A comienzos del año 2009 se sancionó el Decreto 137/09, en el ámbito de la Agencia de Protección Ambiental, con el objetivo de alcanzar "el desarrollo de políticas que permitan evaluar e implementar medidas concretas de adaptación y mitigación frente al cambio climático". Es importante señalar que el Plan nada dice de la calidad del aire, la contaminación por gases tóxicos y la salud de la población. En particular respecto de la incorporación del gas natural como política de mitigación de gases de efecto invernadero, si bien no hay duda de que esta iniciativa se tradujo en una reducción de las emisiones de $\mathrm{CO} 2$, aparentemente aumentarían las emisiones de $\mathrm{CH} 4$ y $\mathrm{N} 2 \mathrm{O}$ en una proporción tal que la mencionada reducción de $\mathrm{CO} 2$ queda neutralizada. Sin embargo, dada la alta incertidumbre en la estimación de las emisiones de $\mathrm{CH} 4$ y N2O producidas por las tecnologías locales (dado que las mediciones son muy escasas y aquellas realizadas presentan una gran variabilidad en los datos obtenidos) resulta imprescindible realizar más estudios de campo específicos que mejoren la precisión de este resultado.
Pero ese mismo año, sectores científicos tanto de la atmósfera como de la salud, junto con diversas ONG, insistieron en jerarquizar el tema de la contaminación atmosférica, incentivando y logrando que el gobierno realizara el cambio de equipos de monitoreo en dos estaciones existentes y que instalara una tercera estación. Como ya se ha mencionado, los nuevos equipos miden monóxido de carbono (CO), óxidos de nitrógeno $(\mathrm{NOx})$ y material particulado $\left(\mathrm{PM}_{10}\right)$. Sin embargo, este logro para el monitoreo de gases contaminantes no resultó en medidas concretas de gestión.

La mayor parte de las políticas implementadas vinculadas ya sea a la gestión del transporte, al manejo de los recursos energéticos o al procesamiento de los desechos urbanos, no estuvieron motivadas en la mejora del ambiente sino en la reducción de costos o en la introducción de nuevas tecnologías originadas en otros países con estándares ambientales más estrictos. Un caso paradigmático es la incorporación del gas natural como combustible en vehículos particulares, de pasajeros, taxis y de carga livianos, que se origina en un exceso de oferta de este combustible y el correspondiente bajo costo. Como excepción se puede mencionar la prohibición de la incineración de residuos sólidos en domicilios, que fue reemplazada por el uso de rellenos sanitarios por Ordenanza Municipal 17.225/73.

El recorrido cronológico de la inclusión del aire en la política pública de la CABA da cuenta del predominio de la agenda internacional por sobre la agenda local. A la vez, también hace visible cómo la presión pública puede influir en la adecuación de algunas respuestas políticas a fin de reducir los niveles de conflicto social más que por reducir los riesgos o las causas de éstos. Ello se evidencia en iniciativas formales que no llegan a implementarse en la práctica.

Por su parte, el monitoreo de la salud aún es débil en la ciudad, especialmente para llevar adelante su correlación con la calidad del aire. Ello devela la vulnerabilidad del sector para incluirse dentro de un sistema integrado de gestión del aire. Por el momento, el seguimiento estadístico de la recolección sistemática de datos sobre eventos de salud relacionados con las enfermedades de notificación obligatoria, la información sobre casos agudos y los decesos, sólo permiten tener un panorama muy general del monitoreo de la salud de la población. 


\section{Vulnerabilidad social y exposición: relaciones con la mortalidad}

La dimensión de impacto del cambio climático y de la contaminación atmosférica vinculada al campo de la salud considera dos factores de riesgo: por un lado el impacto de los cambios de las temperaturas y precipitaciones y por otro, de la contaminación del aire. Ambos temas tienen una larga tradición en la literatura epidemiológica, particularmente focalizados en episodios agudos o intensivos tanto de contaminación como de temperaturas extremas, conocidos por provocar grandes impactos en la salud pública. Sin embargo, en la última década se desarrollaron metodologías que posibilitan el estudio de los efectos de cambios más sutiles en las condiciones ambientales como las variaciones ordinarias en la temperatura o en la contaminación del aire a niveles considerados seguros, variaciones que sin embargo pueden producir un impacto importante en la salud de la población, y la consiguiente sobrecarga crónica sobre las instituciones públicas y privadas del sistema de salud ${ }^{31-34}$.

El SAEMC, a través de un estudio de tipo descriptivo, ecológico, con un enfoque estadístico de series temporales sobre los casos de muertes acaecidas en la CABA durante el período comprendido entre los años 2005 y $2009^{8}$, mostró una clara correlación entre los cambios en las temperaturas y las variaciones en la mortalidad, tanto para la mortalidad total como discriminada por causas cardiovasculares y respiratorias. Las asociaciones fueron más fuertes en verano e invierno, y más leves en las estaciones de transición, primavera y otoño. La mortalidad por causas cardiovasculares aparece más fuertemente correlacionada con los cambios en las temperaturas que la mortalidad por causas respiratorias.

Durante el verano se observa una relación positiva entre el aumento de la temperatura y el aumento en el valor diario de mortalidad, mostrando un aumento de $1,8 \%$ de muertes por cada grado de incremento en la temperatura media registrado el mismo día. Esta correlación se mantiene durante los tres días siguientes, aunque con menor intensidad. Los subgrupos más afectados son las mujeres y los mayores de 64 años. En los meses de invierno, por el contrario, el aumento de la temperatura cumple una función protectora, por lo tanto la correlación es inversa, y su efecto más fuerte se advierte a los 3 y 4 días de producido el aumento, con un $1,1 \%$ menos de muertes diarias.
Estos resultados son coherentes con la literatura reciente que describe los efectos de los cambios en la temperatura sobre la salud humana ${ }^{35-37}$, incluso en ciudades de clima templado como lo es Buenos Aires. De este modo los resultados obtenidos cumplen el propósito postulado por SAEMC en cuanto a proveer información que contribuya al diseño de políticas para controlar los gases de efecto invernadero.

En lo que hace al análisis de contaminación del aire urbano por gases tóxicos, también se corroboró de manera clara la correlación entre la variación de los compuestos del aire medidos y la mortalidad. Se utilizaron los datos sobre concentración de CO y NOX ya que estos son los compuestos medidos por la Red de Monitoreo del GCBA, sin embargo la presencia de estos compuestos en la atmósfera se correlaciona de diversas formas con la de otros no medidos como el material particulado, el Ozono, etc. SAEMC verificó un aumento del 3,6\% de muertes diarias al día siguiente a una suba en 1 ppm (parte por millón) de CO atmosférico. El análisis de los óxidos de nitrógeno (NOx) muestra también una correlación importante con la mortalidad diaria, particularmente debido a causas respiratorias. El mismo día en que aumenta $10 \mathrm{ppb}$ (partes por billón) el nivel de NOx en el aire, se incrementa $0,7 \%$ la mortalidad por esta causa y $0,4 \%$ la mortalidad por causas cardiovasculares.

Los resultados de este trabajo expresan de manera contundente que aún en una ciudad que generalmente no sobrepasa los niveles de concentración de contaminantes establecidos en la normativa local, la contaminación produce una afectación a la salud que en casos extremos desemboca en la muerte.

La exposición a estresores térmicos agrava dolencias existentes, en particular las relacionadas con el aparato respiratorio y cardiovascular, aumentando de esta manera la susceptibilidad de una parte de la población a la contaminación atmosférica. Es decir que ambos procesos se retroalimentan y es muy posible que los efectos combinados del impacto al organismo de las temperaturas y la contaminación sea mayor que la adición simple de los efectos de ambos estresores. Al distribuirse en el tiempo, estas afectaciones a la salud se naturalizan, quedado enmascaradas como "normales", desvinculadas de las condiciones ambientales que las favorecen; en ocasiones la atención que reciben es menor que episodios extraordinarios de índole catastrófica, que sin embargo tienen un efecto epidemiológico menos importante. 


\section{Comentarios finales}

La gestión urbana de la calidad del aire en la CABA viene enfrentando procesos atmosféricos complejos frente a los cuales se diferencian dos tipos de problemáticas. Una de ellas, la más enfatizada en la actualidad, se refiere a los compuestos GEI modificadores del clima de la Tierra. La segunda, que viene siendo relegada, considera aquellos compuestos que afectan a la salud de su población. Ello se pone en evidencia al revisar la cronología de las medidas gubernamentales donde se observa, bajo distintos lineamientos político- económicos, el predominio de la agenda internacional por sobre la agenda local.

Si bien inicialmente el foco de la gestión se puso en la repercusión de las emisiones en la salud, con el correr de los años ese objetivo se fue desdibujando. Y cuando el tema del aire urbano se resituó en la agenda, lo hizo tomando en cuenta gases que no afectaban inmediatamente la salud, sino a través de los efectos del cambio global. En la actualidad la medición de gases tóxicos responde a un programa de gobierno separado al de las mediciones GEI, con el agravante de que esos resultados no están siendo utilizados para mejorar la situación de la salud en la población de la ciudad.

En cuanto a las medidas tomadas para reducir emisiones GEI tanto en el ámbito local como en el nacional, se destaca el incentivo a emplear GNC. Sin embargo, los estudios desarrollados dejaron en evidencia el manto de incertidumbres tanto científicas como políticas, que envuelve dicha cuestión: al analizar el uso de GNC se ha visto que aumentaría la proporción de $\mathrm{CH} 4$ y N2O en las emisiones, neutralizando así la reducción de CO2. El apoyo dado a esta tecnología durante la década de los noventa posiblemente obedeció a la búsqueda de desacople de una parte del consumo de combustible fósil exportable en el conurbano con mayor concentración de población y de vehículos particulares de Argentina, combustible que a la vez resultaba más económico para los particulares. En este sentido las mismas o incluso mayores dudas resultarían del empleo de biocombustibles, cuya adopción se traduciría en una reducción de gases de efecto invernadero. Pero al ser implementada sin las tecnologías adecuadas puede incrementar la emisión de gases tóxicos, repercutiendo negativamente en la salud.

También el análisis del impacto de la contaminación y las temperaturas sobre la población de la CABA ha puesto de manifiesto la existencia de numerosas incertidumbres, confirmando los hallazgos de otros trabajos publicados sobre temas convergentes ${ }^{38}$. Una primera y fundamental incertidumbre se relaciona con el hecho de que, formando parte de una cuenca ambiental amplia que incluye a todo el Área Metropolitana de Buenos Aires, la CABA es el único distrito que cuenta con estadísticas de salud debidamente procesadas y disponibles para estudios de este tipo, mientras que para el resto del área (con aproximadamente diez millones de habitantes) la información se encuentra demasiado agregada o directamente no disponible. Lo mismo sucede con la información sobre la composición del aire: las únicas estaciones de monitoreo existentes que informan datos diarios se localizan en el territorio de la CABA, quedando entonces sin monitoreo una gran parte de la cuenca atmosférica.

No obstante estas dificultades derivadas de la falta o la insuficiencia de datos sistematizados, el diseño del Proyecto SAEMC ha recuperado las estadísticas existentes en los sectores de salud, monitoreo del aire y temperatura, logrando correlacionarlas. Así se obtuvieron resultados significativos que ratifican la exposición de los porteños al riesgo por la contaminación del aire y que, en casos extremos, desemboca en la muerte. Los indicadores de salud utilizados se refieren a registros de mortalidad por ser los más completos; su análisis confirma la necesidad de aplicar medidas de mitigación para reducir la vulnerabilidad de la población frente a emisiones tóxicas.

$\mathrm{Al}$ reflexionar acerca de las iniciativas oficiales, se puede apreciar que los resultados de correlación entre contaminación y muertes pondrían en cuestión los niveles máximos de concentración establecidos en la normativa local y, en consecuencia, sobre los niveles de aceptabilidad del riesgo asumidos por las autoridades relativos a la contaminación del aire. Aunque existen evidencias científico-técnicas de la peligrosidad de los gases contaminantes para la salud, no se implementan mecanismos para reducir la vulnerabilidad de la población frente a estos eventos. Por ello sería importante indagar sobre esta baja percepción de la peligrosidad, expresada concretamente en carencia de políticas para disminuir el riesgo.

De esto se desprende que enfatizar en las medidas de mitigación frente al cambio climático constituye una falsa opción cuando de riesgo social se trata, donde la salud de la población es afectada de manera cotidiana por respirar elevadas concentraciones de gases tóxicos. Ambas situaciones pueden desembocar en catástrofes. Si la contaminación del aire no es debidamente abordada puede causar desastres tanto intensivos 
(por ejemplo, gran número de defunciones e incremento en el número de enfermos luego de una ola de calor, de aumento de partículas por una erupción volcánica o contaminación por quema de biomasa) como extensivos (con menor número de muertos cotidianos pero que sumados en el tiempo dan una cifra mucho más significativa que en un evento intensivo).

A diferencia de los intensivos, los eventos extensivos suelen quedar ocultos en la consideración general. Por ejemplo, las inundaciones catastróficas ocurridas en ciudades argentinas de llanura, como Santa Fe en 2003 (donde los datos oficiales reconocen 23 muertos) o La Plata en 2013 (con más de 50 muertos), han llamado la atención tanto del público como de los medios de comunicación, trayendo a la luz la cuestión del cambio climático y su influencia en el aumento de la frecuencia y severidad de eventos extremos. Pero si el mismo número de personas mueren en una semana debido al aumento de los niveles de contaminación en diferentes momentos y lugares de una ciudad, esto pasa desapercibido, como si se tratara de un hecho normal. Corresponde enfatizar, entonces, que las causas de los desastres intensivos y extensivos están interconectadas y se superponen; por ello deben ser abordados en conjunto para dar cuenta así de toda su complexidad.

La investigación realizada ha permitido identificar el tipo de vacíos de información que requiere ser cubierto como para reducir la incertidumbre en materia de gestión ambiental. Para profundizar en el conocimiento relativo a la peligrosidad de la contaminación del aire, entonces, sería necesario:

- contar con información estadística sobre eventos en salud como los egresos hospitalarios y las consultas de urgencia (que permitirían aumentar la comprensión del impacto de factores ambientales);
- tener registro del contexto estructural y su relación con la mortalidad y morbilidad de los individuos (incluyendo nivel barrial, familiar y/o individual); $\mathrm{y}$

- registrar patrones diferenciales de exposición para poder medir el impacto de las políticas de salud y las respuestas a la exposición a contaminantes del aire en diferentes áreas del territorio.

La importancia de abordar la gestión de la salud y del aire de manera integrada implicaría la implementación de los monitoreos adecuados de la atmósfera y la salud, permitiendo la reducción de las incertidumbres mencionadas. A partir de ello, se podrán conocer las tendencias acerca del incremento o disminución de la contaminación, y de las temperaturas, favoreciendo el diseño y difusión de medidas para contribuir de manera preventiva a la protección de la población.

Los resultados del estudio realizado pusieron de manifiesto que la gestión de la calidad del aire tiene efectos en la gestión de la salud. Conocer estos vínculos propende a un mejor uso de los recursos y eventualmente a una disminución de la vulnerabilidad de los diferentes grupos, a partir de la dotación de recursos de información, prevención, atención médica, etc. Al mismo tiempo, alertan para no adoptar de manera a-crítica modelos, protocolos y mediciones y externos a la propia realidad.

Finalmente es necesario resaltar los alcances de esta perspectiva centrada en el riesgo y la incertidumbre en los términos ya planteados, lo que ha permitido superar comunicaciones parciales provenientes de los campos disciplinarios involucrados, para lograr una lectura abarcativa del problema del aire en su conjunto sin perder las especificidades de cada dimensión pero articulándolas en beneficio de procesos preventivos.

\section{Colaboradores}

Todas las autoras han colaborado en la redacción final del texto. L Davidowski, R Abrutzky y A. Murgida realizaron la investigación que sustenta los resultados. La primera aportó conocimientos sobre aspectos físico-químicos del aire, R. Abrutzky sobre los aspectos sociales y efectos sobre la salud y A Murgida, el análisis histórico e institucional. Finalmente, CE Natenzon aportó la concepción del artículo, basado en sus trabajos teóricos sobre riesgo ambiental. 


\section{Agradecimientos}

Los resultados que aquí se presentan contaron con financiamiento de la NSF-National Science Foundation para el proyecto "South American Emissions Mega Cities and Climate" - SAEMC; y con fondos que el CONICET-Consejo Nacional de Investigaciones Científicas y Técnicas, otorgó al Proyecto. Se reconoce el apoyo brindado por la Universidad de Buenos Aires mediante el subsidio a la investigación UBACyT. Las autoras agradecen los oportunos comentarios de Carlos Machado de Freitas al texto inicial.

\section{Referencias}

1. Bell ML, Davis DL, Gouveia N, Borja-Arbuto VH, Cifuentes LA. The avoidable health effects of air pollution in three Latin American cities. Environmental Research 2005; 100:431-440.

2. Cakmak S, Dales RE, Vidal CB. Air pollution and mortality in Chile: Susceptibility among the elderly. Environmental Health Perspectives 2007; 115(4):524-527.

3. Sánchez H, Albala C. Desigualdades en salud: adultos en comunas del Gran Santiago. Rev. Médica de Chile 2004; 132(4):453-460.

4. Sanhueza P, Pizarro J, Vargas C, Torreblanca M, Passalacqua $\mathrm{M}$. Health risk estimation due to carbon monoxide pollution at different spatial levels in Santiago, Chile. Environmental Monitoring and Assessment 2010; 167:165-173.

5. Amarillo A, Carreras H. The effect of airborne particles and weather conditions on pediatric respiratory infections in Cordoba, Argentine. Environmental Pollution 2012; 170:217-221.

6. D’Angiola A, Dawidowski L, Gómez D, Osses M. Onroad traffic emissions in a megacity. Atmospheric Environment 2010; 44(4):483-493.

7. Gallardo L, Escribano G, Dawidowski L, Rojas N, Andrade M, Osses M. Evaluation of vehicle emission inventories for carbon monoxide and nitrogen oxides for Bogotá, Buenos Aires, Santiago, and São Paulo. Atmospheric Environment 2012; 47:12-19.

8. Abrutzky R, Dawidowski L, Matus P, Romero Lankao P. Health Effects of Climate and Air Pollution in Buenos Aires: A First Time Series Analysis. Journal of Environmental Protection 2012; 3:262-271.

9. Abrutzky R, Ibarra S, Matus P, Romero Lankao P, Pereyra V, Dawidowski L (). Atmospheric pollution and mortality. A comparative study between two Latin American cities: Buenos Aires (Argentina) and Santiago (Chile). International Journal of Environment and Health 2013; 6(4):363-380.

10. Natenzon CE, Marlenko N, González S, Ríos D, Murgida A, Meconi G, Calvo A. Las dimensiones del riesgo en ámbitos urbanos. Catástrofes en el Area Metropolitana de Buenos Aires. En: Alessandri Carlos AF, Bertoncello R, compiladores Procesos territoriales en Argentina y Brasil. Buenos Aires, São Paulo: Universidad de Buenos Aires, Universidad de San Pablo; 2003. p. 255-276.

11. Filgueira C. Estructura de oportunidades y vulnerabilidad social. Aproximaciones conceptuales recientes. Politica y Gestión 2006; 9:18-64.

12. Kristie EBI, McGregor G. Mudanças climáticas, ozônio troposférico e partículas suspensas: impactos na saúde. Cien Saude Colet 2009; 14(6):2281-2293.

13. Giddens A. Consecuencias de la modernidad. Madrid: Alianza; 1993.

14. Beck U. La sociedad del riesgo. Hacia una nueva modernidad. Barcelona: Paidós; 1992.

15. Lavell A. Degradación ambiental, riesgo y catástrofe urbano. Problemas y conceptos: hacia la definición de una agenda de investigación. En: Fernández MA, compiladora. Ciudades en riesgo. Degradación ambiental, riesgos urbanos y catástrofes en América Latina. Lima: La Red; 1996. p. 21-60. 
16. Funtowicz S, De Marchi B. Ciencia posnormal, complejidad reflexiva y sustentabilidad. En: Leff E, coordinador. La Complejidad Ambiental. México: PNUMA, UNAM, Siglo XXI; 2000. p. 54-84.

17. Natenzon CE. Catástrofes naturales, riesgo e incertidumbre. Buenos Aires: FLACSO; 1995. (Serie Documentos e Informes de Investigación, n. 197)

18. Herzer H, Rodríguez C, Celis A, Bartolomé M, Caputo G. Convivir con el riesgo o la gestión del riesgo. Buenos Aires: Centro; 2002. Mimeo.

19. Blaikie PM, Cannon T, Davis I, Wisner B. At Risk: Natural Hazards, People's Vulnerability and Disasters. London: Routledge; 1994.

20. Funtowicz S, Ravetz J. Riesgo global, incertidumbre e ignorancia. Epistemología política. Ciencia con la gente. Buenos Aires: CEAL; 1993. p. 11-42.

21. Instituto Nacional de Estadística y Censos (INDEC). Censo Nacional de Población y Viviendas. 2010. [accesado 2014 jul 7]. Disponible en: http://www.indec.gov.ar

22. Nabel PE, Becerra Serial R. Uso de técnicas geomáticas para el mapeo y caracterización de unidades geomórficas de la Ciudad de Buenos Aires (Argentina) mediante interpretación visual de parámetros morfométricos. Boletín de la Sociedad Geológica Mexicana 2007; 60(2):173-185.

23. Camilioni I, Barros V. Unidades temáticas Clima y Cambio Climático. Atlas Ambiental de Buenos Aires. CONICET/ ANPCYT/ MACN/ FADU-UBA/ GCBA. [accesado 2014 jul 7]. Disponible en: http://www.atlas debuenosaires.gov.ar

24. Rusticucci M. Impacto del cambio climático global. Estado de Situación. Encrucijadas 2007; 41. [accesado 2014 jul 7]. Disponible en: http://www.uba.ar/encrucijadas /41/sumario/enc41-estadodesituacion.php.

25. Menéndez A, Re M. Características hidrológicas de los ríos Paraná y de la Plata. Vulnerabilidad de la Zona Costera. Informe final. Argentina $2^{\circ}$ Comunicación de Cambio Climático; 2005. p. 49-74.

26. Clichevsky N. Pobreza y políticas urbano-ambientales en Argentina. Santiago de Chile: Cepal; 2002.

27. Korc ME. Situación de los programas de gestión de calidad del aire urbano en América Latina y El Caribe. Lima: Cepis; 2000.

28. Murgida AM, Guebel CF, Natenzon CE. Frasco L. El aire en la agenda pública: el caso de la Ciudad Autónoma de Buenos Aires. En: Sánchez Rodríguez R, editor Respuestas urbanas al cambio climático en América Latina. Santiago de Chile: Cepal; 2013. p. 137-157.

29. Ravella OR, Karol JL, Aón LC. Transporte y ambiente: utopías urbanas, ciudades reales, ciudades posibles. Transporte y Territorio 2012; 6:27-51.

30. Gobierno de la Ciudad Autónoma de Buenos Aires. Informe Anual Ambiental 2008. [accesado 2014 jul 7]. Disponible en: http://www.buenosaires.gob.ar/areas/med ambiente/informe_anual_08.php?menu_id=30025.

31. Braga ALF, Zanobetti A, Schwartz J. The Lag Structure Between Particulate Air Pollution and Respiratory and Cardiovascular Deaths in 10 US Cities. J Occup Environ Med 2001; 43(11):662-667.
32. Braga ALF, Zanobetti A, Schwartz J. The Effect of Weather on Respiratory and Cardiovascular Deaths in 12 U.S. Cities. Environ Health Perspect 2002; 110(9): 759-767.

33. Curtis L, Rea W, Smith-Willis P, Fenyves E, Pan Y. Adverse health effects of outdoor air pollutants. Environ Int 2006; 32(6):815-830

34. Stieb DM, Judek S, Burnett RT. Meta-Analysis of Time-Series Studies of Air Pollution and Mortality: Effects of Gases and Particles and the Influence of Cause of Death, Age, and Season. Journal of the Air \& Waste Management Association 2002; 52(4):470-484.

35. McMichael J, Woodruff RE, Hales S. Climate change and human health: present and future risks. The Lancet 2006; 367(9513):859-869.

36. O'Neill M, Zanobetti A, Schwartz J. Modifiers of the Temperature and Mortality Association in Seven US Cities. Am J Epidemiol 2003; 157(12):1074-1082.

37. Patz JA, Engelberg D, Last J. The effects of changing weather on public health. Annu Rev Public Health 2000; 21:271-307.

38. Clougherty JE, Kubzansky LD. A framework for examining social stress and susceptibility to air pollution in respiratory health. Cien Saude Colet 2010; 15(4):20592074.

Artigo apresentado em 03/04/2014

Aprovado em 10/06/2014

Versão final apresentada em 12/06/2014 